\title{
Effects of exercise training on autonomic modulation and mood symptoms in patients with obstructive sleep apnea
}

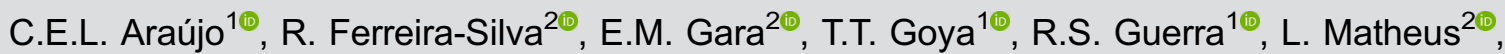 \\ E. Toschi-Dias ${ }^{1 \oplus}$, A.G. Rodrigues ${ }^{1 \oplus}$, E.R.F. Barbosa ${ }^{1 \oplus}$, R. Fazan $\mathrm{Jr}^{3 \oplus}$, G. Lorenzi-Filho $^{1 \oplus}$, \\ C.E. Negrão ${ }^{1,40}$, and L.M. Ueno-Pardi ${ }^{1,20 \ltimes}$ \\ ${ }^{1}$ Instituto do Coração, Hospital das Clinicas, Faculdade de Medicina, Universidade de São Paulo, São Paulo, SP, Brasil \\ ${ }^{2}$ Escola de Artes, Ciências e Humanidades, Universidade de São Paulo, São Paulo, SP, Brasil \\ ${ }^{3}$ Departamento de Fisiologia, Faculdade de Medicina de Ribeirão Preto, Universidade de São Paulo, Ribeirão Preto, SP, Brasil \\ ${ }^{4}$ Escola de Educação Física e Esportes, Universidade de São Paulo, São Paulo, SP, Brasil
}

\begin{abstract}
We evaluated the effects of exercise training (ET) on the profile of mood states (POMS), heart rate variability, spontaneous baroreflex sensitivity (BRS), and sleep disturbance severity in patients with obstructive sleep apnea (OSA). Forty-four patients were randomized into 2 groups, 18 patients completed the untrained period and 16 patients completed the exercise training (ET). Beat-to-beat heart rate and blood pressure were simultaneously collected for 5 min at rest. Heart rate variability (RR interval) was assessed in time domain and frequency domain (FFT spectral analysis). BRS was analyzed with the sequence method, and POMS was analyzed across the 6 categories (tension, depression, hostility, vigor, fatigue, and confusion). ET consisted of 3 weekly sessions of aerobic exercise, local strengthening, and stretching exercises (72 sessions, achieved in $40 \pm 3.9$ weeks). Baseline parameters were similar between groups. The comparisons between groups showed that the changes in apnea-hypopnea index, arousal index, and $\mathrm{O}_{2}$ desaturation in the exercise group were significantly greater than in the untrained group $(P<0.05)$. The heart rate variability and BRS were significantly higher in the exercise group compared with the untrained group $(P<0.05)$. ET increased peak oxygen uptake $(P<0.05)$ and reduced $P O M S$ fatigue $(P<0.05)$. A positive correlation $(r=0.60, P<0.02)$ occurred between changes in the fatigue item and OSA severity. ET improved heart rate variability, BRS, fatigue, and sleep parameters in patients with OSA. These effects were associated with improved sleep parameters, fatigue, and cardiac autonomic modulation, with ET being a possible protective factor against the deleterious effects of hypoxia on these components in patients with OSA.
\end{abstract}

Key words: Exercise; Cardiac autonomic modulation; Spontaneous baroreflex sensitivity; Mood symptoms; Obstructive sleep apnea

\section{Introduction}

Obstructive sleep apnea (OSA) is characterized by recurrent decreases (hypopneas) or complete cessations (apneas) of airflow during sleep in the presence of a continued respiratory effort (obstructive airflow events) due to collapse of the upper airway, which leads to oxygen desaturations, arousals, and sleep fragmentation (1).

Repeated events of hypoxia and reoxygenation lead to an increase of reactive oxygen species (2), insulin resistance (3), systemic inflammation, vascular dysfunction, and increase in sympathetic nervous activity (4), placing OSA patients at risk for cardiovascular disorders and neuropsychological symptoms that include mood changes (5). These changes in patients with OSA lead to impaired daytime performance (6) and increased occupational and motor vehicle accidents (7), which translate into difficulties in social adjustment related to fatigue $(8,9)$.

OSA patients have a cardiac autonomic dysfunction, including depressed baroreflex sensitivity $(10,11)$, diminished vagal tone, and exaggerated cardiac sympathetic responsiveness $(12,13)$. Previous studies also show that adults with mood disturbances experience reduced baroreflex sensitivity and autonomic modulation $(14,15)$. Reduced heart rate variability (HRV) has been documented as a predictor of cardiovascular risk and mortality (16), suggesting that the presence of cardiac autonomic 
dysfunction has a significant impact on the prognosis of patients with OSA.

Accumulated evidence over the past decade shows that exercise training (ET) plays a role in OSA management. Several randomized controlled trials have demonstrated that ET reduces apnea-hypopnea index (AHI) $(17,18)$ and improves objective and subjective sleep quality $(17)$ independently of weight loss $(17,18)$.

Additionally, chronic aerobic exercise can be an efficient treatment against mood disturbance compared with other interventions (19). Aerobic exercise combined with medication also improves mood disturbance, and this strategy is more efficient than medication alone (20). Few studies have investigated the effect of ET on the autonomic nervous system and mood status in patients with OSA. Therefore, the aim of this study was to investigate the effects of supervised ET on autonomic modulation and mood status in patients with OSA without other major comorbidities.

\section{Material and Methods}

\section{Patients}

Men and women aged 40 to 65 years who underwent conventional nocturnal polysomnography at the Sleep Laboratory of the Heart Institute, School of Medicine, University of São Paulo were selected for the present study. These individuals were part of a large study regarding ET on cerebral function in patients with OSA. Patients taking any medication, who had body mass index $(\mathrm{BMI}) \geqslant 40 \mathrm{~kg} / \mathrm{m}^{2}$, hypertension, cardiopulmonary disease, chronic kidney disease, diabetes mellitus, atrial fibrillation, arrhythmia, pacemaker, kidney failure, echocardiographic evidence of impaired left ventricular function (ejection fraction $<45 \%$ ), history of psychiatric disorders or other neurodegenerative disorders, smoking or alcohol abuse (2 or more drinks per day), any sleep apnea treatment, sleep disorders other than OSA, and circadian desynchrony (eg, shift workers) were excluded from the study.

Forty-four patients who were newly diagnosed with OSA ( $\mathrm{AHI}>15$ events per hour of sleep) were included in the study. They were randomly assigned on a 1:1 basis to the untrained group $(n=22)$ and exercise-trained group $(n=22)$. All patients were sedentary adults who had not exercised regularly for at least 3 months before enrolling in this study. All non-menopausal women were studied between the first and the fifth day after the onset of menstruation. All patients underwent echocardiographic evaluation at baseline. Full nocturnal polysomnography and maximal exercise capacity were performed at baseline and at the end of the study. The patients selected for the untrained group were asked to maintain their physical activity habits during the study. No patient in the untrained group reported changes in physical activity when questioned. This study was approved by the Research
Committee of the Heart Institute (SDC 3536/10/125) and the Human Subject Protection Committee at the Clinical Hospital of the School of Medicine of the University of São Paulo (0833/10). The study is registered at Clinical Trials.gov (NIH: NCT002289625). All patients gave written informed consent to participate in the study. Full nocturnal polysomnography, echocardiographic measurements, maximal exercise capacity, cardiac autonomic evaluation, and a profile of mood states were performed at baseline and at the end of the study.

\section{Sleep analysis}

OSA evaluation was performed according to the method previously described (21). All participants underwent a nocturnal polysomnography (Embla N7000, Medcare Flaga, Iceland). Monitoring included collection of electroencephalograms, electrooculograms, submental and tibial electromyography signals, airflow measurements (oronasal thermistor and pressure cannula), and movement of the chest and abdominal cords by bracing, measurement of oxyhemoglobin saturation by oximetry, and electrocardiography (ECG). Obstructive sleep apnea events were defined as $90 \%$ or greater reduction in respiratory amplitude for at least $10 \mathrm{~s}$ with thoracoabdominal effort. Sleep recordings were scored according to the criteria of Rechtschaffen and Kales (22). Hypopneas were defined as a $50 \%$ or greater reduction in respiratory range for at least $10 \mathrm{~s}$ associated with oxygen desaturation $\geqslant 3 \%$ and/or arousal. Individuals with $>70 \%$ obstructive events were defined as individuals with OSA. The AHI was defined as the number of apnea and hypopnea events per hour of sleep. Sleep stages were classified according to the criteria of the American Academy of Sleep Medicine (23).

\section{Blood pressure}

Clinical blood pressure (BP) readings were obtained from the left arm of subjects while seated, after $5 \mathrm{~min}$ of quiet rest, with a mercury sphygmomanometer. All subjects had at least 3 BP measurements obtained on separate occasions taken by one investigator. Systolic and diastolic BP was recorded at the first appearance and disappearance (phases I and V, respectively) of Korotkoff sounds. The subjects were classified as normotensive if the average systolic and diastolic BP levels were $\leqslant 140$ or $90 \mathrm{mmHg}$, respectively.

\section{Profile of Mood States - POMS}

The Profile of Mood States - POMS (24) scale has been one of the instruments most used to assess emotional and mood states as well as the variation of emotions associated with exercise and psychological wellbeing. The POMS version used in this research corresponded to a reduced version of the original scale. The validity design of this POMS version, as well as its internal consistency for Portuguese was published 
elsewhere (25). Briefly, the reduced version consists of a list of 42 adjectives for patients to describe themselves during the preceding week using a 5-point Likert scale format. Standard scoring of the POMS yields a global distress score referred to as total mood disturbance as well as scores for 6 subscales: Tension-Anxiety, Depression-Dejection, Anger-Hostility, Vigor-Activity, FatigueInertia, and Confusion-Bewilderment.

\section{Cardiac function}

Left ventricular function was assessed by an ultrasound device (Vivid E9, GE Healthcare, USA) using a 4-MHz multifrequency transducer. Left ventricular volumes and left ventricular ejection fraction were analyzed using 2-dimensional 2-chamber and 4-chamber examination by the Simpson's method. All study subjects underwent echocardiography performed by an echocardiographer blinded to the patient's history, who only was aware of the polysomnography before monitoring. All echocardiograms were performed, analyzed, and reported by the same echocardiographer, whose objective was to exclude the presence of structural heart disease.

\section{Cardiopulmonary exercise testing}

Cardiorespiratory capacity was measured by a cardiopulmonary maximal exercise test on an electromagnetically braked cycle ergometer (Medifit 400L, Medical Fitness Equipment, The Netherlands), using a ramp protocol with workload increments of 10 or $15 \mathrm{~W}$ every minute at $60 \mathrm{rpm}$ up to exhaustion. Oxygen uptake $\left(\mathrm{VO}_{2}\right)$ and carbon dioxide production were determined by means of gas exchange on a breath-by-breath basis in a computerized system (SensorMedics, Vmax 229 model, USA). Anaerobic threshold was determined to occur at the breakpoint between the increase in the carbon dioxide production and $\mathrm{VO}_{2}$ (V-slope) or the point at which the end-tidal oxygen partial pressure curves reached its minimum value and began to rise. Respiratory compensation point was determined at the point at which the ventilatory equivalent for carbon dioxide was lowest before a systematic increase and when end-tidal carbon dioxide partial pressure reached a maximum and began to decrease. Peak $\mathrm{VO}_{2}$ was defined as the maximum attained $\mathrm{VO}_{2}$ at the end of the exercise period in which the subject could no longer maintain the cycle ergometer velocity at $60 \mathrm{rpm}$.

\section{Experimental protocol}

The study was performed in a quiet, temperaturecontrolled room $\left(21\right.$ to $22^{\circ} \mathrm{C}$ ), in the morning at approximately the same time in the pre- and post-intervention with the subjects in the supine position. All signals were collected during $5 \mathrm{~min}$ of the subject being in the supine position with normal quiet breathing. Heart rate was continuously collected through lead II of an ECG recorded on a computer using the Windaq program (Dataq
Insturments, USA) at a frequency of $1000 \mathrm{~Hz}$. Simultaneously, arterial BP was monitored using a Finometer $\mathrm{PRO}^{\mathbb{R}}$ (Finapress Medical System, The Netherlands), which provides noninvasive measurement on beat-tobeat basis. Respiratory rate was monitored using a piezoelectric thoracic belt (Pneumotrace II, model 1132, Respiration Transducer, UFI, USA) placed around the upper abdomen. All signals were recorded on a computer at a frequency of $1000 \mathrm{~Hz}$ and then analyzed in the Windaq program.

\section{Spontaneous baroreflex sensitivity analysis}

For the analysis of baroreflex function and HRV, ECG and arterial pressure recordings were processed by customized computer software to generate series of successive values of RR-intervals $(R R)$ and systolic arterial pressure (SAP).

Spontaneous baroreflex control (BRS) was analyzed by sequence method, as proposed by Bertinieri and co-workers (26). This method assumes that spontaneous arterial pressure ramps elicit baroreflex-mediated responses in RR length (27). Series of SAP were searched for increasing or decreasing ramps with at least 3 values of arterial pressure. When a pressure ramp (up or down) shows linear correlation $(r>0.8)$ with RR changes at the same direction, a baroreflex sequence has been found. The spontaneous BRS is considered as the slope $(\mathrm{ms} / \mathrm{mmHg})$ of the linear regression between SAP and RR. The baroreflex gain of each subject is taken as the average slope, calculated from all the sequences.

\section{Heart rate variability analysis}

Cardiac autonomic modulation was assessed from spontaneous fluctuations in the time series of RR. All the series were visually inspected, and artifacts or transients were manually removed. For time domain analysis we calculated the standard deviation of RR values (SDNN) and the root mean square of successive differences between normal heart beats (RMSSD). For frequency domain analysis of HRV, beat-by-beat series of RRs were converted to an evenly spaced series using cubic spline interpolation $(10 \mathrm{~Hz})$ and divided into half-overlapping sequential sets of 512 data points (Welch periodogram). Segments with transients that could affect the calculation of power spectral density were excluded. A Hanning window was used to attenuate side effects, and the spectrum of each segment was calculated by fast Fourier transform (FFT). The spectra of RR were integrated and quantified in the very-low frequency (VLF: $<0.04 \mathrm{~Hz}$ ), lowfrequency range (LF: 0.04 to $<0.15 \mathrm{~Hz}$ ), and in the highfrequency range (HF: 0.15 to $0.4 \mathrm{~Hz}$ ). The absolute values of the RR interval spectral densities are reported in absolute values $\left(\mathrm{ms}^{2}\right)$ and are also reported in normalized units (nu) as previously recommended for interpretation of spectral HRV indices in sleep research (28). The normalization procedure was performed by dividing the power of 
the LF or HF component by the total spectral power from which the power of the very low frequency (VLF) component had been subtracted. The LF/HF ratio was also calculated. Respiratory rate was measured in breaths per minute to certify that respiratory frequency of each subject was into the high-frequency range, i.e., between 0.15 and $0.4 \mathrm{~Hz}$.

\section{Exercise training protocol}

The supervised exercise training consisted of 72 exercise sessions at a frequency of 3 days per week. Each exercise session had a total duration of $60 \mathrm{~min}$ and consisted of 5 min of stretching the upper and lower limbs, $40 \mathrm{~min}$ of aerobic exercise on a cycle ergometer (in the first month for $30 \mathrm{~min}$ ), $10 \mathrm{~min}$ of local strengthening exercises, and $5 \mathrm{~min}$ of muscle stretching (21). The intensity of aerobic exercise was determined by the corresponding heart rate of the anaerobic threshold and the respiratory compensation point (detected from the cardiorespiratory capacity test). Patients in the untrained group were told not to engage in systematic physical activity programs during the control period.

\section{Statistical analysis}

The data are reported as means $\pm S D$ (range) and proportions (gender). Unpaired Student's $t$-test was used to compare differences between groups (age, BMI, metabolic and functional parameters, baseline cardiovascular parameters, sleep parameters, POMS). Analysis of variance with repeated measures was used to compare within and between group differences in BMI, metabolic and functional parameters, cardiovascular parameters, sleep parameters, and POMS, before and after intervention. In the case of significance, post-hoc comparisons were performed with the Duncan test. $\mathrm{P}<0.05$ was considered statistically significant. All analyses were

Table 1. Baseline physical characteristics, sleep parameters, and cardiac autonomic modulation of participants with obstructive sleep apnea in the untrained and exercise-trained groups.

\begin{tabular}{|c|c|c|c|}
\hline Characteristics & $\begin{array}{c}\text { Untrained } \\
\quad(n=18)\end{array}$ & $\begin{array}{c}\text { Exercise-trained } \\
\quad(n=16)\end{array}$ & $P$ \\
\hline Gender, M/F & $12 / 6$ & $6 / 10$ & 0.17 \\
\hline Age, years & $54 \pm 8$ & $50 \pm 6$ & 0.20 \\
\hline Weight, kg & $80 \pm 14$ & $79 \pm 14$ & 0.85 \\
\hline $\mathrm{BMI}, \mathrm{kg} / \mathrm{m}^{2}$ & $29 \pm 4$ & $30 \pm 4$ & 0.48 \\
\hline $\mathrm{SBP}, \mathrm{mmHg}$ & $123 \pm 11$ & $129 \pm 15$ & 0.90 \\
\hline DBP, $\mathrm{mmHg}$ & $79 \pm 9$ & $79 \pm 6$ & 0.92 \\
\hline $\mathrm{VO}_{2}$ peak, $\mathrm{mL} \cdot \mathrm{kg}^{-1} \cdot \mathrm{min}^{-1}$ & $26 \pm 6$ & $22 \pm 5$ & 0.10 \\
\hline \multicolumn{4}{|l|}{ Polysomnography } \\
\hline $\mathrm{AHI}$, events/h & $45 \pm 27$ & $44 \pm 31$ & 0.91 \\
\hline $\mathrm{O}_{2}$ desaturation, events $/ \mathrm{h}$ & $34 \pm 27$ & $38 \pm 31$ & 0.69 \\
\hline Total arousal, events & $216 \pm 121$ & $195 \pm 114$ & 0.45 \\
\hline \multicolumn{4}{|l|}{ Autonomic parameters } \\
\hline $\mathrm{RR}, \mathrm{ms}$ & $942 \pm 129$ & $891 \pm 135$ & 0.26 \\
\hline SDNN, $\mathrm{ms}^{2}$ & $49 \pm 15$ & $43 \pm 23$ & 0.72 \\
\hline RMSSD, $\mathrm{m}^{2}$ & $40 \pm 19$ & $31 \pm 23$ & 0.23 \\
\hline BRS gain, $\mathrm{ms} / \mathrm{mmHg}$ & $10 \pm 4$ & $8 \pm 5$ & 0.45 \\
\hline $\mathrm{LF}, \mathrm{ms}^{2}$ & $606 \pm 508$ & $1161 \pm 2097$ & 0.31 \\
\hline $\mathrm{HF}, \mathrm{ms}^{2}$ & $658 \pm 717$ & $711 \pm 1063$ & 0.87 \\
\hline$L F, n u$ & $52 \pm 19$ & $56 \pm 19$ & 0.60 \\
\hline $\mathrm{HF}, \mathrm{nu}$ & $48 \pm 19$ & $44 \pm 19$ & 0.60 \\
\hline $\mathrm{LF} / \mathrm{HF}$ & $1.5 \pm 1$ & $1.8 \pm 2$ & 0.47 \\
\hline
\end{tabular}

Data are reported as means $\pm S D$, except gender (n). There were no significant differences between the untrained and exercise-trained groups ( $t$-test and chisquared test (gender)). M: male; F: female; BMI: body mass index; SBP: systolic blood pressure; DBP: diastolic blood pressure; $\mathrm{VO}_{2}$ peak: oxygen uptake at peak exercise; $\mathrm{AHI}$ : apnea-hypopnea index; $\mathrm{O}_{2}$ : oxygen; RR: ECG RR interval; SDNN: mean standard deviation of the RR intervals; RMSSD: root mean square of the differences between consecutive RR intervals; BRS: spontaneous baroreflex sensitivity; LF: low frequency band; HF: high frequency band; nu: normalized units. 
performed using STATISTICA 12 software (StatSoft Inc., $\mathrm{OK})$.

\section{Results}

Ten patients were excluded from the study. In the untrained group, 2 patients were excluded from the

Table 2. Baseline profile of mood states (POMS) subscales scores in patients with obstructive sleep apnea.

\begin{tabular}{lccc}
\hline & Untrained $(n=18)$ & Exercise-trained $(n=16)$ & $P$ \\
\hline Tension & $8 \pm 6$ & $9 \pm 6$ & 0.17 \\
Depression & $3 \pm 5$ & $3 \pm 3$ & 0.20 \\
Hostility & $4 \pm 5$ & $4 \pm 4$ & 0.85 \\
Force & $11 \pm 5$ & $11 \pm 5$ & 0.98 \\
Fatigue & $4 \pm 6$ & $7 \pm 5$ & 0.07 \\
Confusion & $5 \pm 2$ & $5 \pm 2$ & 0.92 \\
\hline
\end{tabular}

Data are reported as means $\pm S D$. There were no significant differences between the untrained and exercise-trained groups ( $t$-test). analysis because they had artifacts in the collection signal, 1 patient started using the continuous positive airway pressure device (CPAP) during the study period, and 1 patient did not complete the exam protocols and was therefore excluded. In the exercise group, 1 patient was excluded because they had artifacts in the collection signal, 3 had inadequate breathing patterns for analysis, and 2 abandoned the physical training.

The baseline characteristics of the untrained and exercise-trained groups are shown in Table 1. No significant differences were observed between groups in age, weight, $\mathrm{BMI}$, systolic $\mathrm{BP}$, diastolic $\mathrm{BP}$, and $\mathrm{VO}_{2}$ peak. There was also no significant difference in baseline sleep and autonomic modulation parameters between groups $(P>0.05)$. Baseline POMS score was also similar between the groups regarding the 6 subscales (Table 2).

\section{Effects of exercise training}

ET attendance varied among patients. Seventy-two sessions or $100 \%$ of ET as planned was achieved in $40 \pm 3$ weeks. The control group was paired with the ET group. ET and clinical follow-up caused no significant

Table 3. Effects of physical training on physical and physiological parameters of participants with obstructive sleep apnea.

\begin{tabular}{|c|c|c|c|c|c|c|}
\hline & \multicolumn{3}{|c|}{ Untrained } & \multicolumn{3}{|c|}{ Exercise-trained } \\
\hline & Pre & Post & Delta & Pre & Post & Delta \\
\hline Weight, kg & $80 \pm 14$ & $81 \pm 14$ & $0.9 \pm 2$ & $79 \pm 14$ & $78 \pm 12$ & $-1.0 \pm 4$ \\
\hline BMI, $\mathrm{kg} / \mathrm{m}^{2}$ & $29 \pm 4$ & $29 \pm 4$ & $0.1 \pm 1$ & $30 \pm 4$ & $30 \pm 4$ & $-0.3 \pm 2$ \\
\hline $\mathrm{SBP}, \mathrm{mmHg}$ & $123 \pm 11$ & $123 \pm 15$ & $-0.2 \pm 14$ & $129 \pm 15$ & $115 \pm 12$ & $-8 \pm 13$ \\
\hline $\mathrm{DBP}, \mathrm{mmHg}$ & $79 \pm 9$ & $77 \pm 8$ & $-2 \pm 13$ & $79 \pm 6$ & $77 \pm 6$ & $-1 \pm 6$ \\
\hline $\mathrm{VO}_{2}$ peak, $\mathrm{mL} \cdot \mathrm{kg}^{-1} \cdot \mathrm{min}^{-1}$ & $26 \pm 6$ & $25 \pm 7$ & $-0.6 \pm 1$ & $22 \pm 5$ & $27 \pm 6^{* \dagger}$ & $5 \pm 3^{\dagger \dagger}$ \\
\hline \multicolumn{7}{|l|}{ Sleep parameters } \\
\hline TTS, min & $395 \pm 45$ & $400 \pm 47$ & $4 \pm 39$ & $366 \pm 59$ & $370 \pm 56$ & $4 \pm 64$ \\
\hline$A H I$, events $/ \mathrm{h}$ & $45 \pm 27$ & $49 \pm 31$ & $4 \pm 10$ & $44 \pm 31$ & $38 \pm 23^{*}$ & $-5 \pm 15^{\dagger \dagger}$ \\
\hline $\mathrm{O}_{2}$ desat, events $/ \mathrm{h}$ & $34 \pm 27$ & $43 \pm 30$ & $9 \pm 20$ & $38 \pm 31$ & $35 \pm 22$ & $-3 \pm 13^{\dagger \dagger}$ \\
\hline Total arousal/events & $216 \pm 121$ & $222 \pm 128$ & $6 \pm 40$ & $195 \pm 114$ & $155 \pm 69^{* \dagger}$ & $-38 \pm 66^{\dagger \dagger}$ \\
\hline Lowest $\mathrm{O}_{2}$ sat, $\%$ & $82 \pm 7$ & $81 \pm 6$ & $-1 \pm 3$ & $78 \pm 10$ & $79 \pm 9$ & $0.5 \pm 4$ \\
\hline \multicolumn{7}{|l|}{ HRV parameters } \\
\hline $\mathrm{RR}, \mathrm{ms}$ & $942 \pm 125$ & $917 \pm 127$ & $-32 \pm 85$ & $891 \pm 135$ & $932 \pm 113^{\dagger}$ & $56 \pm 106^{\dagger \dagger}$ \\
\hline $\mathrm{SDNN}, \mathrm{ms}^{2}$ & $49 \pm 15$ & $48 \pm 25$ & $-1.6 \pm 20$ & $43 \pm 23$ & $50 \pm 22$ & $6.9 \pm 16^{\dagger \dagger}$ \\
\hline RMSSD, $\mathrm{ms}^{2}$ & $40 \pm 19$ & $34 \pm 22$ & $-6 \pm 20$ & $31 \pm 23$ & $36 \pm 22$ & $5 \pm 13^{\dagger \dagger}$ \\
\hline BRS gain, $\mathrm{ms} / \mathrm{mmHg}$ & $10 \pm 4$ & $8 \pm 4$ & $-2 \pm 4$ & $8 \pm 5$ & $10 \pm 6^{* \dagger}$ & $2 \pm 3^{\dagger \dagger}$ \\
\hline LF abs, $\mathrm{ms}^{2}$ & $606 \pm 508$ & $915 \pm 1322$ & $343 \pm 877$ & $1161 \pm 2097$ & $750 \pm 972$ & $-410 \pm 168$ \\
\hline $\mathrm{HF}$ abs, $\mathrm{ms}^{2}$ & $658 \pm 717$ & $643 \pm 775$ & $22 \pm 617$ & $711 \pm 1063$ & $669 \pm 715$ & $-42 \pm 728$ \\
\hline LF nu & $52 \pm 19$ & $57 \pm 14$ & $8 \pm 18$ & $56 \pm 19$ & $54 \pm 22$ & $-3 \pm 21$ \\
\hline HF nu & $48 \pm 19$ & $43 \pm 15$ & $-2 \pm 16$ & $44 \pm 9$ & $47 \pm 22$ & $3 \pm 21$ \\
\hline $\mathrm{LF} / \mathrm{HF}$ & $1.5 \pm 1$ & $1.6 \pm 1$ & $0.13 \pm 1$ & $1.8 \pm 2$ & $2.0 \pm 2$ & $0.05 \pm 2$ \\
\hline
\end{tabular}

Data are reported as means $\pm S D$. ${ }^{*} P<0.05$, compared to untrained in post-intervention (between groups); ${ }^{\dagger} \mathrm{P}<0.05$ compared to pre-intervention (within group); ${ }^{\dagger \dagger} \mathrm{P}<0.05$ compared to the delta of the untrained group (repeated measures ANOVA). Pre: baseline measurement; Post: final measurement. SBP: systolic blood pressure; DBP: diastolic blood pressure; $\mathrm{VO}_{2}$ peak: oxygen uptake at peak exercise; TTS: total sleep time; AHI: apnea-hypopnea index; $\mathrm{O}_{2}$ desat: oxygen desaturation; $\mathrm{O}_{2}$ sat: oxygen saturation; HRV: heart rate variability; RR: ECG RR interval; SDNN: mean standard deviation of the RR intervals; RMSSD: root mean square of the differences between consecutive RR intervals; BRS: spontaneous baroreflex sensitivity; LF: low frequency in band; HF: high frequency band; nu: normalized units. 
changes in body weight, BMI, and blood pressure (Table 3). After the intervention, a significant increase in $\mathrm{VO}_{2}$ peak was observed only in the ET group $(P<0.05)$. A significant reduction in arousal was observed in the trained group $(\mathrm{P}<0.05)$. Comparisons between groups showed that the changes in $\mathrm{AHI}, \mathrm{O}_{2}$ desaturation, and total arousals were significantly higher in the exercise group $(P<0.05)$. The RR interval increased significantly after training. Gain of BRS was significantly higher in the exercise group compared with the untrained group $(P<0.05)$. Furthermore, analysis of changes of $R R$,

Table 4. Effects of physical training on the profile of mood state score in patients with moderate to severe sleep apnea.

\begin{tabular}{lrrrrr}
\hline & \multicolumn{2}{c}{ Untrained } & & \multicolumn{2}{c}{ Exercise-trained } \\
\cline { 2 - 3 } \cline { 5 - 6 } & Pre & Post & & Pre & Post \\
\hline Tension & $8 \pm 6$ & $7 \pm 4$ & & $9 \pm 6$ & $6 \pm 6$ \\
Depression & $3 \pm 5$ & $2 \pm 2$ & & $3 \pm 3$ & $2 \pm 3$ \\
Hostility & $4 \pm 5$ & $3 \pm 3$ & & $4 \pm 4$ & $2 \pm 3$ \\
Force & $11 \pm 5$ & $12 \pm 5$ & & $11 \pm 5$ & $14 \pm 5$ \\
Fatigue & $4 \pm 6$ & $6 \pm 5$ & & $7 \pm 5$ & $3 \pm 4^{\star \dagger}$ \\
Confusion & $5 \pm 2$ & $4 \pm 2$ & & $5 \pm 2$ & $4 \pm 2$ \\
\hline
\end{tabular}

Data are reported as means $\pm S D$. ${ }^{*} \mathrm{P}<0.05$, compared to untrained in post-intervention (between groups), ${ }^{\dagger} \mathrm{P}<0.05 \mathrm{com}-$ pared to pre-intervention (within group) (repeated measures ANOVA). Pre: baseline measurement; Post: final measurement.
SDNN, RMSSD, and BRS were higher in the trained than in the untrained group $(P<0.05)$.

Concerning the POMS scale, there was a significant reduction in fatigue $(P<0.05)$ only in the exercise group (Table 4). Between-group comparisons showed the changes (Figure 1) in fatigue were significantly higher in the exercise group compared with untrained group $(P<0.05)$. There was a positive correlation $(r=0.60, P=0.02)$ between fatigue and $\mathrm{AHI}$.

\section{Discussion}

The main and new findings of the study are that ET in patients with OSA i) improved HRV and baroreflex function and ii) improved fatigue in the mood profile.

In previous studies, patients with OSA exhibited reduced baroreflex sensitivity and impaired autonomic modulation to the heart $(15,29)$. The present study reveals that exercise training improved BRS in patients with OSA. Stiffening of large cardiothoracic arteries leads to a lower afferent trigger for a given change in $\mathrm{BP}$, which reduces the afferent response capacity of the baroreceptors (30). Intermittent hypoxia and reoxygenation is a potential atherogenic factor, leading to damage of the wall of large arteries. A previous study found that middle-aged patients with severe OSA, without overt cardiovascular diseases, demonstrate early signs of atherosclerosis by means of increased arterial stiffness and carotid remodeling (31). ET may improve vascular

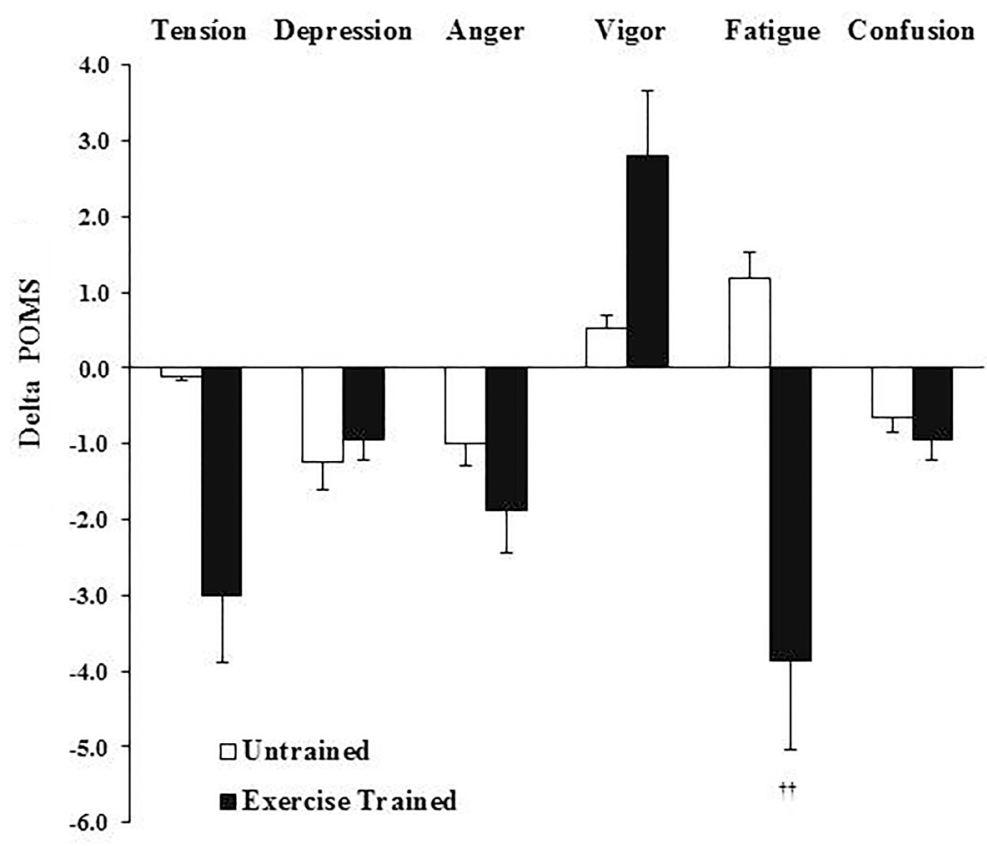

Figure 1. Delta of mood profile changes in patients with obstructive sleep apnea after intervention. Data are reported means $\pm S D$. ${ }^{\dagger \dagger} \mathrm{P}<0.05$ vs Untrained (t-test between groups). POMS: Profile of Mood States. 
structural change. Recently, Guerra et al. (21) showed that ET promoted a marked increase in forearm blood flow at rest and during handgrip exercise in patients with moderate to severe OSA (21).

It is possible that both mechanical and neural components play important roles in BRS regulation. The rapid heart period responses to baroreflex engagement in humans are predominantly mediated through efferent vagal activity (32). The present study shows that $\mathrm{RR}$ interval (index of vagal activity) was significantly increased by ET. The mechanisms involved in the improvement of HRV and BRS were not the scope of the present study. However, we can suggest that the improvement in BRS was due to an increased vascular structure, as well as endothelial factors (through the release of vasodilating substances). These responses ameliorate the vascular properties and, in consequence, facilitate the BRS $(30,33)$.

Our results are in line with a previous study (34) that measured overnight HRV using a Holter ECG before and after 9 months of ET in patients with moderate OSA. The authors found that the RR interval increased in the exercise-trained group, whereas the control group had decreased parasympathetic and overall HRV parameters. It is possible that the effects of ET on autonomic modulation may extend even into the nocturnal period. In the present study, the improvement in cardiorespiratory capacity together with higher parasympathetic predominance may attenuate effects of hypoxiarelated neural, vessel wall properties, and mechanical changes in the heart that lead to a decrease in BRS. The higher parasympathetic modulation in the exercisetrained group may also be a cardioprotective factor that contributes to the increasing of the fibrillation threshold as well as greater BP buffering action, including those caused by intermittent hypoxia.

A possible explanation for improving sleep parameters, more specifically those changes in AHI, arousal index, and $\mathrm{O}_{2}$ desaturation in the exercise-trained group, was due to the fact that ET would attenuate the narrowing or collapse of the upper airways by the redistribution of rostral fluid in the neck, reducing the severity of OSA (35). Alternatively, the increase in muscle tone of the genioglossus (36) reduces hypoxia and arousal episodes during sleep even without a significant change in body weight $(21,35)$.

Fatigue is a common complaint in patients with OSA (37). A previous study found a wide range of fatigue in patients with OSA, including approximately $42 \%$ reporting a significant amount of fatigue (38). Fatigue is very prevalent in conditions associated with obesity, psychological distress, or in conditions of insufficient physical activity (39). Fatigue encompasses physical and psychological factors, and like pain, fatigue is a symptom that is almost always assessed by self-report. In this investigation, the fatigue-inertia category item of POMS included signs and symptoms of burnout, exhaustion, low energy level, and tiredness. ET significantly reduced the fatigue-inertia category item of the mood profile in patients with moderate to severe OSA. In the present study, the greater reduction in $\mathrm{AHI}$ and sleep fragmentation in the ET patients contributed to stabilization of the sleep structure, restoring correct levels of oxyhemoglobin in the blood and decreasing respiratory effort leading to a more restorative sleep that may affect the fatigue factor. The positive correlation $(r=0.60, P=0.02)$ between the delta fatigue item and the delta $\mathrm{AHI}$ in the ET group strengthen this idea. These findings were consistent with a previous investigation in which improvement in fatigue after CPAP treatment was reported (40).

The improvement in OSA severity with ET might have contributed to the reduction in fatigue in patients with OSA and likely to the improvement in stressful daily routine. Because fatigue is also reported as a risk factor for injuries in work accidents (8) or risk of car accidents (7), the significant reduction in fatigue with ET can minimize the risk for these dangerous situations in these patients. Further studies on the mechanism of improvement in cardiac autonomic modulation and fatigue with ET in patients with OSA are needed.

Seventy-two ET sessions, conducted 3 times a week for 6 months as planned were achieved by some patients. In fact, 72 exercise sessions varied in frequency ( 1 to 3 ) over a period of $40 \pm 3$ weeks, which occurs in real life during human intervention protocols. Despite the limitation, ET was able to significantly improve autonomic modulation of heart rate, baroreflex sensitivity, fatigue, and sleep parameters in patients suffering with OSA.

Among patients with OSA and no comorbidities, supervised ET induced adaptations in cardiac autonomic functions, baroreflex sensitivity, and symptoms of fatigue. These effects are associated with greater cardioprotection and improvement in well-being in patients with moderate to severe OSA.

\section{Acknowledgments}

The authors are grateful to the polysomnography technicians at the Heart Institute's Sleep Laboratory for performing the over night polysomnography and for the excellent patient care. This study was supported by FAPESP (São Paulo Research Foundation; grant \#2010/ 15064-6) to L.M. Ueno-Pardi. C.E. Negrão was supported by Conselho Nacional de Pesquisa (CNPq; grant \#303573/2015-5). 


\section{References}

1. Epstein LJ, Kristo D, Strollo PJ Jr, Friedman N, Malhotra A Patil SP, et al. Clinical guideline for the evaluation, management and long-term care of obstructive sleep apnea in adults. J Clin Sleep Med 2009; 5: 263-276, doi: 10.5664/ jcsm.27497.

2. McGown AD, Makker H, Elwell C, Al Rawi PG, Valipour $A$, Spiro SG. Measurement of changes in cytochrome oxidase redox state during obstructive sleep apnea using near-infrared spectroscopy. Sleep 2003; 26: 710-716, doi: 10.1093/sleep/ 26.6.710.

3. McArdle N, Hillman D, Beilin L, Watts G. Metabolic risk factors for vascular disease in obstructive sleep apnea. $A m$ J Respir Crit Care Med 2007; 175: 190-195, doi: 10.1164/ rccm.200602-2700C.

4. Ueno-Pardi LM, Guerra RS, Goya TT, Silva RF, Gara EM, Lima MF, et al. Muscle metaboreflex control of sympathetic activity in obstructive sleep apnea. Med Sci Sports Exerc 2017; 49: 1424-1431, doi: 10.1249/MSS.0000000000001242.

5. Macey PM, Woo MA, Kumar R, Cross RL, Harper RM. Relationship between obstructive sleep apnea severity and sleep, depression and anxiety symptoms in newly-diagnosed patients. PLoS One 2010; 5: e10211, doi: 10.1371/ journal.pone.0010211.

6. Cheshire K, Engleman H, Deary I, Shapiro C, Douglas NJ. Factors impairing daytime performance in patients with sleep apnea/hypopnea syndrome. Arch Intern Med 1992; 152: 538-541, doi: 10.1001/archinte.1992.00400150068012.

7. Martiniuk AL, Senserrick T, Lo S, Williamson A, Du W, Grunstein RR, et al. Sleep-deprived young drivers and the risk for crash: the DRIVE prospective cohort study. JAMA Pediatr 2013; 167: 647-655, doi: 10.1001/jamapediatrics. 2013.1429.

8. Swaen GMH, van Amelsvoort LG, Bültmann U, Kant I. Fatigue as a risk fator for being injured in an occupational accident: results from the Maastricht Cohort Study. Occup Environ Med 2003; 60: i88-i92, doi: 10.1136/oem.60. suppl_1.i88.

9. Kazemeini E, Braem MJ, Moorkens G, Balina S, Kastoer C, Op de Beeck $S$, et al. Scoring of hypersomnolence and fatigue in patients with obstructive sleep apnea treated with a titratable custom-made mandibular advancement device. J Clin Sleep Med 2019; 15: 623-628, doi: 10.5664/jcsm.7728.

10. Carlson JT, Hedner JÁ, Sellgren J, Elam M, Wallin BG. Depressed baroreflex sensitivity in patients with obstructive sleep apnea. Am J Respir Crit Care Med 1996; 154: 14901496, doi: 10.1164/ajrccm.154.5.8912770.

11. Lombardi C, Pengo MF, Parati G. Obstructive sleep apnea syndrome and autonomic dysfunction. Auton Neurosci 2019; 221: 102563, doi: 10.1016/j.autneu.2019.102563.

12. Harper RM, Kumar R, Macey PM, Ogren JA, Richardson $\mathrm{HL}$. Functional neuroanatomy and sleep-disordered breathing: implications for autonomic regulation. Anat $\operatorname{Rec} 2012$; 295: 1385-1395, doi: 10.1002/ar.22514.

13. Sequeira VCC, Bandeira PM, Azevedo JCM. Heart rate variability in adults with obstructive sleep apnea: a systematic review. Sleep Sci 2019; 12: 214-221, doi: 10.5935/19840063.20190082.
14. Virtanen R, Jula A, Salminen JK, Voipio-Pulkki LM, Helenius $\mathrm{H}$, Kuusela T, et al. Anxiety and hostility are associated with reduced baroreflex sensitivity and increased beat-to-beat blood pressure variability. Psychosom Med 2003; 65: 751756, doi: 10.1097/01.PSY.0000088760.65046.CF.

15. Toschi-Dias E, Trombetta IC, da Silva VJ, Maki-Nunes C, Alves MJ, Angelo LF, et al. Symptoms of anxiety and mood disturbance alter cardiac and peripheral autonomic control in patients with metabolic syndrome. Eur J Appl Physiol 2013; 113: 671-679, doi: 10.1007/s00421-012-2476-8.

16. Bigger JT Jr, Fleiss JL, Steinman RC, Steinman RC. Frequency domain measures of heart period variability and mortality after myocardial infarction. Circulation 1992; 85: 164-171, doi: 10.1161/01.CIR.85.1.164.

17. Kline CE, Crowley EP, Ewing GB, Burch JB, Blair SN, Durstine $\mathrm{JL}$, et al. The effect of exercise training on obstructive sleep apnea and sleep quality: a randomized controlled trial. Sleep 2011; 34: 1631-1640, doi: 10.5665/ sleep.1422.

18. Berger M, Barthelemy JC, Hupin D, Raffin J, Dupré C, Labeix $\mathrm{P}$, et al. Benefits of supervised community physical activity in obstructive sleep apnoea. Eur Respir J 2018; 52 : 1801592, doi: 10.1183/13993003.01592-2018.

19. Carneiro LSF, Fonseca AM, Vieira-Coelho MA, Mota MP, Vasconcelos-Raposo J. Effects of structured exercise and pharmacotherapy vs. pharmacotherapy for adults with depressive symptoms: a randomized clinical trial. J Psychiatr Res 2015; 71: 48-55, doi: 10.1016/j.jpsychires.2015. 09.007

20. Moura AMS, Lamego MK, Paes F, Rocha NBF, SimoesSilva V, Rocha AS, et al. Comparison among aerobic exercise and other types of interventions to treat depression: a systematic review. CNS Neurol Disord Drug Targets 2015; 14: 1171-1183, doi: 10.2174/1871527315 666151111120714

21. Guerra RS, Goya TT, Silva RF, Lima MF, Barbosa ERF, Alves MJNN, et al. Exercise training increases metaboreflex control in patients with obstructive sleep apnea. Med Sci Sports Exerc 2019; 51: 426-435, doi: 10.1249/MSS.0000 000000001805.

22. Rechtschaffen A, Kales A. A manual of standardized terminology, techniques, and scoring system for sleep stages of human subjects. Bethesda: National Institute of Neurological Disease and Blindness; 1968.

23. Iber C, Ancoli-Israel S, Chessonn A, Quan SF. The AASM manual for the scoring of sleep and associated events: rules, terminology and technical specifications. 1st ed. Westchester: American Academy of Sleep Medicine; 2007.

24. Norcross JC, Guadagnoli E, Prochaska JO. Factor structure of the Profile of Mood States (POMS): two partials replications. J Clin Psychol 1984; 40: 1270-1277, doi: 10.1002/ 1097-4679(198409)40:5<1270::AID-JCLP2270400526>3.0. $\mathrm{CO} ; 2-7$

25. Viana MF, Almeida PL, Santos RC. Adaptação portuguesa da versão reduzida do Perfil de Estados de Humor: POMS [in Portuguese]. Aná Psicológica 2001; 19: 77-92. 
26. Bertinieri G, di Rienzo M, Cavallazzi A, Ferrari AU, Pedotti A, Mancia G. A new approach to analysis of the arterial baroreflex. J Hypertens Suppl 1985; 3: S79-S81.

27. Silva LEV, Dias DPM, da Silva CAA, Salgado HC, Fazan R Jr. Revisiting the sequence method for baroreflex analysis. Front Neurosci 2019; 13: 17, doi: 10.3389/fnins.2019.00017.

28. Burr RL. Interpretation of normalized spectral heart rate variability indices in sleep research: a critical review. Sleep 2007; 30: 913-919, doi: 10.1093/sleep/30.7.913.

29. Urbanik D, Gac P, Martynowicz H, Poręba M, Podgórski $M$, Negrusz-Kawecka M, et al. Obstructive sleep apnea as a predictor of reduced heart rate variability. Sleep Med 2019; 54: 8-15, doi: 10.1016/j.sleep.2018.09. 014.

30. Ueno LM, Hamada T, Moritani T. Cardiac autonomic nervous activities and cardiorespiratory fitness in older men. J Gerontol A Biol Sci Med Sci 2002; 57: M605-M610, doi: 10.1093/gerona/57.9.M605.

31. Drager LF, Bortolotto LA, Lorenzi MC, Figueiredo AC, Krieger EM, Lorenzi-Filho G, et al. Early signs of atherosclerosis in obstructive sleep apnea. Am J Respir Crit Care Med 2005; 172: 613-618, doi: 10.1164/rccm.2005033400C.

32. Hunt BE, Fahy L, Farquhar WB, Taylor JA. Quantification of mechanical and neural components of vagal baroreflex in humans. Hypertension 2001; 37: 1362-1368, doi: 10.1161/ 01.HYP.37.6.1362.

33. Ueno LM, Miyachi M, Matsui T, Takahashi K, Yamazaki K, Hayashi K, et al. Effect of aging on carotid artery stiffness and baroreflex sensitivity during head-out water immersion in man. Braz J Med Biol Res 2005; 38: 629-637, doi: 10.1590/S0100-879X2005000400018.

34. Berger M, Raffin J, Pichot V, Hupin D, Garet M, Labeix P, et al. Effect of exercise training on heart rate variability in patients with obstructive sleep apnea: a randomized controlled trial. Scand J Med Sci Sports 2019; 29: 12541262, doi: $10.1111 / \mathrm{sms} .13447$.

35. Kline CE. Exercise: shifting fluid and sleep apnoea away. Eur Respir J 2016; 48: 23-25, doi: 10.1183/13993003. 00797-2016.

36. Giebelhaus V, Strohl KP, Lormes W, Lehmann M, Netzer N. Physical exercise as an adjunct therapy in sleep apnea An open trial. Sleep Breath 2000; 4: 173-176.

37. Bardwell WA, Moore P, Ancoli-Israel S, Dimsdale JE. Fatigue in obstructive sleep apnea: driven by depressive symptoms instead of apnea severity? Am J Psychiatry 2003; 160: 350-355, doi: 10.1176/appi.ajp.160.2.350.

38. Mills PJ, Kim JH, Bardwell W, Hong S, Dimsdale JE. Predictors of fatigue in obstructive sleep apnea. Sleep Breath 2008; 12: 397-399, doi: 10.1007/s11325-008-0192-8.

39. Vgontzas AN, Bixler EO, Chrousos GP. Obesity-related sleepiness and fatigue: the role of the stress system and cytokines. Ann N Y Acad Sci 2006; 1083: 329-344, doi: 10.1196/annals.1367.023.

40. Yu BH, Ancoli-Israel S, Dimsdale JE. Effect of CPAP treatment on mood states in patients with sleep apnea. J Psychiatr Res 1999; 33: 427-432, doi: 10.1016/S00223956(99)00020-5. 